\title{
3D Printing and Ophthalmology for the Community
}

\section{Sheng Chiong Hong}

Ophthalmology Registrar, Gisborne Hospital, Ormond Road, 4010, Gisborne, New Zealand

*Corresponding author: Hong Sheng Chiong, Ophthalmology Registrar, Gisborne Hospital, Ormond Road, 4010, Gisborne, New Zealand, Tel: 64 6-869 0500; E-mail: hschiong@gmail.com

Rec date: May 26, 2015; Acc date: May 28, 2015; Pub date: May 30, 2015

Copyright: (C) 2015 Hong SC. This is an open-access article distributed under the terms of the Creative Commons Attribution License, which permits unrestricted use, distribution, and reproduction in any medium, provided the original author and source are credited.

Keywords: $3 \mathrm{D}$ printing in ophthalmology; Smartphone teleophthalmology; Smartphone retinal camera; Smartphone slit lamp microscope

\section{Editorial}

People may have heard of three dimensional (3D) printed firearms, which carries bad publicity for 3D printing, yet amazing things have been achieved with simple desktop 3D printers within the medical field. 3D printing was almost unheard of until the last 5 years; as key additive-manufacturing patents expire, affordable desktop 3D printers mushroomed globally.

The technology of 3D printing is not new. Actually, the idea can be traced back to as early as the 1890s when Blanther suggested a layered method for making a mould for topographical relief maps [1]. However, it was not until 1987 when the first additive manufacturing machine emerged [2]. In simpler terms, 3D printing is in fact $2 \mathrm{D}$ printing but over and over again, layered together. As patents expire, innovation took over the stage and people started building affordable, user-friendly $3 \mathrm{D}$ printers that are now widely available. Fused deposition modelling (FDM) printers are the most common consumer oriented printers and brands such as MakerBot and Ultimaker currently dominate the consumer market. These are low end 3D printers that have made the biggest impacts and allowed innovative leaps in the medical field. Recently, an interventional radiologist managed to save a patient's spleen with 3D printing technology [3]. The patient was diagnosed with a number of complicated tortuous splenic aneurysms and conventionally, she would have been treated with splenectomy. However, the interventional radiologist managed to spare her spleen by coiling the aneurysms instead. The success was not by luck or co-incidence; it was through countless practices on a $3 \mathrm{D}$ printed replica of the patient's splenic aneurysms.

$3 \mathrm{D}$ printing in ophthalmology is an exciting field. There are a variety of uses ranging from optics industry printing spectacles to scientist printing ocular tissues such as sclera and cornea [4]. 3D printing has the advantage of low-cost yet high-level customization, making it cost-effective for making one of a kind devices or prostheses. Combined with 3D scanning technology, a patient could have his or her customized prosthetic eye $3 \mathrm{D}$ printed in a matter of days instead of weeks.

Ophthalmology is a highly specialized field, and many primary care physicians feel uncomfortable managing patients with eye problems. A cross-sectional survey questionnaire study conducted in Canada revealed that $80 \%$ of family medicine resident doctors felt "somewhat comfortable" or "not comfortable at all" in managing ophthalmological conditions [5]. General practitioners often seek advice or consultation from ophthalmologists. However, conventional referral methods such as telephone calls and fax are not efficient.
Describing an ocular condition in words over the phone by a nonophthalmology physician can be difficult. A photograph of the condition is worth a thousand words; unfortunately, ocular imaging devices such as the fundus camera and slit lamp microscope are expensive, with price ranges out of reach by most general practices.

Too often in my practice I see patients present late with terrible eye conditions due to delayed treatment secondary to incorrect diagnosis in the first instance. Although common things are common, inappropriate diagnosis made in the primary care setting has not been infrequent. For example, microbial conjunctivitis is the most common diagnosis that has been given to patients with a red eye. However, there are many other common causes of red eyes such as keratitis, allergic conjunctivitis, blepharitis, anterior uveitis, acute angle closure glaucoma, corneal abrasion and subconjunctival haemorrhage. One particular example, a patient presented to her family doctor with a painful red eye associated with mild photophobia was told that she had conjunctivitis and treated with a course of topical antibiotic ointment. Her symptoms worsened and she represented to her family doctor again five days later. This time, she was given a different antibiotic eye drop. Two days later, she presented to the emergency department with worsening of persistent symptoms. Eventually, I saw her in my eye clinic and slit lamp examination revealed an obvious dendritic ulcer on her corneal surface. The most likely culprit is herpes simplex virus, not microbial conjunctivitis. Unfortunately, she suffered unnecessarily from delayed treatment secondary to incorrect diagnosis and management. In addition, she was unable to work during that period; the economic cost of missed and wrong diagnosis is huge.

To solve these problems, my team and I have been working on an open-source ophthalmic equipment project. By using a 3D printer and a smartphone, we are able to produce a variety of diagnostic and imaging ophthalmic equipments at a cost at least a hundred times cheaper than conventional equipment. We have recently described a $3 \mathrm{D}$ printable retinal imaging device (Figure 1) that can be used in conjunction with a smartphone to photograph the retina [6]. To make it accessible globally, the blueprint files of the device are made freely available online so any clinician could download and print the device for clinical examination. By applying the same principle, we have also produced a slit lamp microscope adapter for smartphones (Figure 2). This device can be used for image-documentation of anterior segment ocular pathologies. Common conditions such as cataract, uveitis, corneal epithelial defect or ulcers can be detected with ease. Instead of spending thousands of dollars on ocular imaging devices, these tools cost less than a hundred dollars. Primary care physicians can now photograph the pathology and send it off for consultation with ease. 
Page 2 of 2

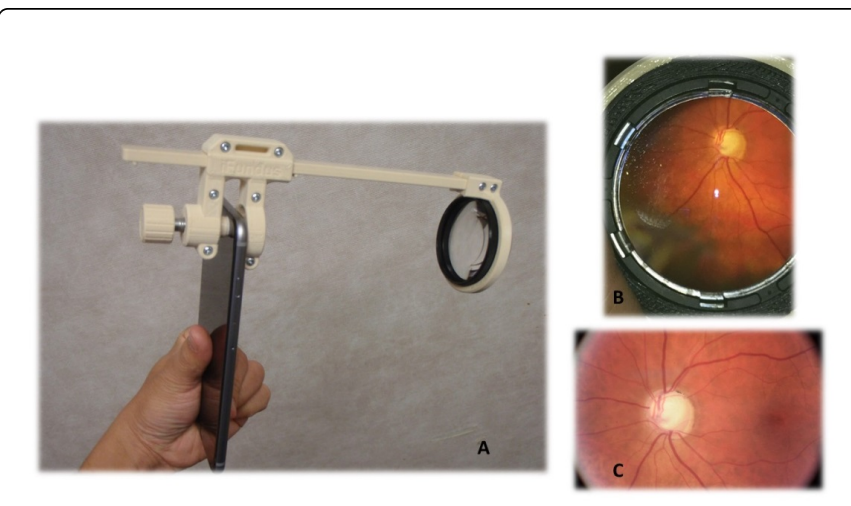

Figure 1: (A) 3D printed retinal imaging adapter on a smartphone. (B) An image of a glaucomatous disc captured with the smartphone retinal imaging adapter. (C) An image of the same glaucomatous disc captured with a standard fundus camera

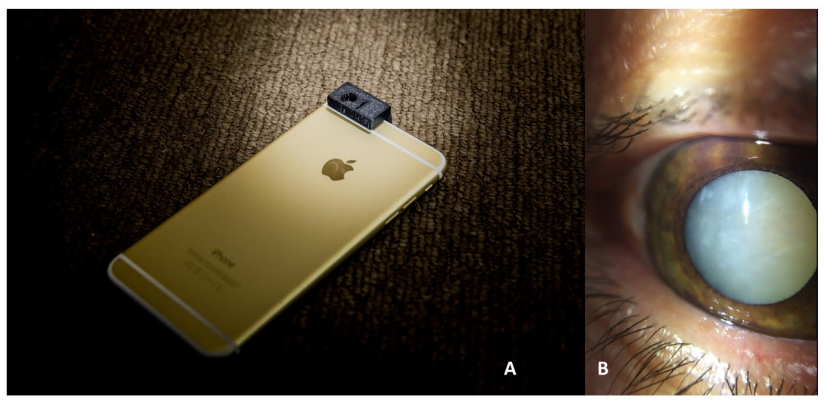

Figure 2: (A) 3D printed smartphone slit lamp microscope. (B) An image of a patient with a white cataract captured on a smartphone with the $3 \mathrm{D}$ printed slit lamp microscope.
$3 \mathrm{D}$ printing has made it possible to create and deliver these equipments in ways that can never be achieved with tradition methods. With the 3D printable files easily transferrable in electronic form, the devices can be emailed to any corner of the world and locally printed in a matter of hours. This may well be the solution to the difficulties faced by healthcare providers in resource limited regions in obtaining affordable eye examination tools. It is essentially a decentralisation process to improve the overall care quality of community ophthalmology.

\section{Acknowledgement}

I would like to thank Dr. Graham Wilson for his clinical supervision, and Dr. Mimi Chiu for providing the photograph in Figure 2A.

\section{References}

1. Bourell DLD, Beaman JJ, Leu MC, Rosen DW (2009) A brief history of additive manufacturing and the 2009 roadmap for additive manufacturing: looking back and looking ahead. US-Turkey Work.

2. Wohlers T, Gornet T (2011) History of additive manufacturing Introduction of non-SL systems Introduction of low-cost 3D printers. Wohlers Rep 2011: 1-23.

3. Itagaki MW (2015) Using 3D printed models for planning and guidance during endovascular intervention: a technical advance. Diagnostic Interv Radiol.

4. Schubert C, van Langeveld MC, Donoso LA (2014) Innovations in 3D printing: a 3D overview from optics to organs. Br J Ophthalmol 98: 159-161.

5. Chan TY, Rai AS, Lee E, Glicksman JT, Hutnik CM (2011) Needs assessment of ophthalmology education for primary care physicians in training: comparison with the International Council of Ophthalmology recommendations. Clin Ophthalmol 5: 311-319.

6. Hong SC (2015) 3D printable retinal imaging adapter for smartphones could go global. Graefes Arch Clin Exp Ophthalmol. 